\title{
Il congresso internazionale di Niagara Falls
}

\author{
Daniela Ducci \\ Segretario del Comitato Italiano IAH \\ daniela@unina.it
}

Il Congresso internazionale IAH del 2012 si è tenuto a Niagara Falls (Canada) (Fig.1) dal 16 al 21 settembre. Ha visto un elevatissimo numero di partecipanti provenienti da 60 paesi (quasi 900), in maggioranza canadesi (470), ma con una folta rappresentanza europea, con l'Italia seconda subito dietro alla Germania come numero di partecipanti (più di 20). Il congresso IAH è stato davvero impeccabile sotto ogni aspetto: ben preparate e gestite le sessioni orali ed eccellenti le sessioni poster. Il tutto accompagnato da keynote lectures di grande rilievo (John Cherry, Allan Freeze, Frank Schwartz e Joszef Tóth) tenutesi nella great hall del Conference Centre, a chiusura di lunches esemplari sia per la qualità del cibo e del servizio, ma soprattutto per la autorevolezza dei convitati. In tale ambito il gruppo degli idrogeologi italiani si è caratterizzato, non solo per l'armonia e compattezza (vedi foto), ma anche per l'elevato livello tecnico dei lavori che hanno presentato nelle varie sessioni. Si tenga conto inoltre che quasi la metà era costituita da giovani (molti dei quali precari della ricerca), che si sono distinti per la qualità delle presentazioni (Fig.2).

Tra le varie sessioni, di particolare spicco quelle su: cambiamenti climatici, karst, acquiferi fessurati, processi nel non saturo, inquinamento, protezione delle risorse idriche sotterranee, acquiferi salini, interazioni acque superficiali/sotterranee, traccianti e isotopi, modellistica, geotermia. Da segnalare infine alcune sessioni decisamente innovative come quelle sull'idrogeologia sostenibile', sui modelli 3D e sulle nanoparticelle. Il terzo giorno del Congresso è stato dedicato alle visite sul campo, che sono riuscite ad conciliare problematiche scientifiche (es. il Karst della Penisola del Niagara) e tecniche (es. la gestione delle discariche in presenza di falde superficiali) in cornici naturalistiche assolutamente indimenticabili, culminanti nelle cascate del Niagara.

Ma Il Congresso internazionale di Niagara Falls non è stato solo ricerca: anche nella "comunità italiana" c'era la presenza del mondo professionale e, proprio in questo contesto, gli stands tecnici di Niagara Falls hanno esibito strumentazioni all'avanguardia, che in taluni casi aprono interessanti nuove prospettive nel mondo dell'idrogeologia

Durante il Congresso, nell'ambito del quale la comunità italiana ha stretto anche i contatti con i nuovi vertici, come il Presidente Ken Howards, si sono riunite le varie Commissioni e Networks IAH, i cui resoconti, redatti dagli italiani che vi hanno partecipato, sono pubblicati sul sito www.iahitaly.it.

Il Comitato Italiano IAH, riunitosi proprio a Niagara Falls, ha in tale sede approvato l'attivazione di un gruppo di lavoro sull'idrogeologia e l'uso sostenibile delle risorse geotermiche sul territorio nazionale (IDROGEOTER), con particolare riguardo agli studi idrogeologici inerenti lo sfruttamento di risorse a "bassa entalpia".

Tale gruppo di lavoro, aperto a tutti gli interessati che abbiano esperienza nel settore, si pone come obiettivo primario di rappresentare un nucleo di riferimento per gli studi idrogeologici nel settore della geotermia. Inoltre si prefigge di lavorare in sinergia con gli Enti con competenze sul territorio in materia geotermica (Ministero per lo Sviluppo Economico, ISPRA, CNR, Enti Locali, ecc.) ed altre organizzazioni (es. UGI), per una programmazione degli studi idrogeologici finalizzati alla gestione sostenibile dell'uso della risorsa geotermica.

Per pervenire a tali obiettivi è prevista anche una attività di divulgazione e diffusione di competenze, soprattutto per la formazione di professionisti, tecnici ed amministratori. Inoltre ci si prefigge di organizzare workshop scientifici con finalità di studio, confronto e discussione e di divulgare i vari steps di lavoro via WEB.

Le attività al momento ipotizzate dal gruppo di lavoro sono:

- analisi dello stato dell'arte nell'utilizzo del potenziale geotermico in sistemi geotermici, sia sul territorio nazionale, che a scala internazionale;

- censimento dei dati, degli studi e delle applicazioni geotermiche in Italia, con particolare riferimento a quelle a bassa entalpia $\left(\mathrm{T}<90^{\circ}\right)$ individuate dal Ministero per lo Sviluppo Economico;

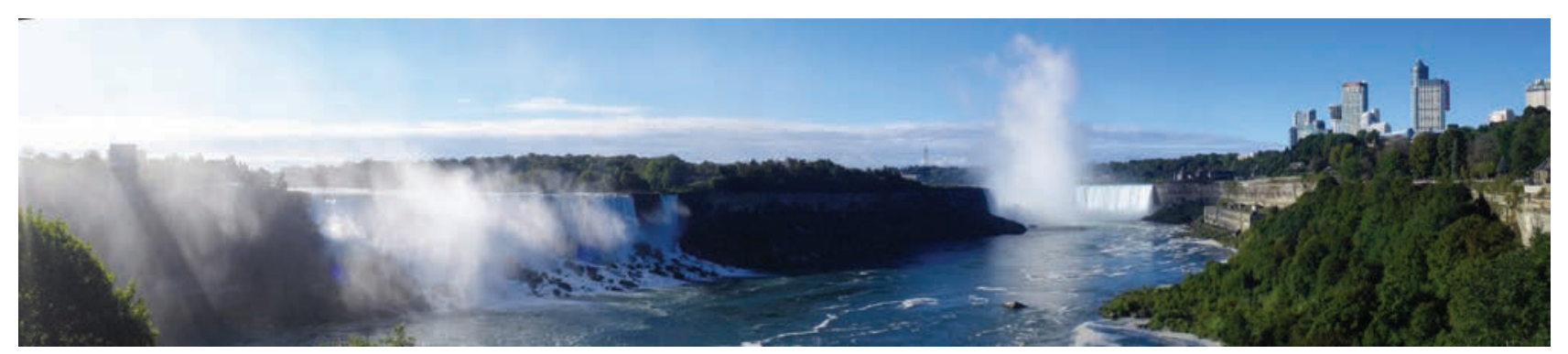

Fig. 1 - Il contesto unico della location del convegno alle Niagara Falls (foto di Marco Pola). 
- analisi del quadro programmatico, normativo e legislativo, a livello internazionale, nazionale (in particolare D. Lgs. n. 22/10 e D. Lgs. n. 28/11) e regionale;

- contributo determinante della geologia e competenza esclusiva dell'idrogeologo nella redazione di studi idrogeotermici regionali e della cartografia del potenziale geotermico;

- criteri ed indirizzi per studi idrogeotermici di dettaglio: analisi e modelli di bilancio idrogeologico, caratterizzazione quali-quantitativa delle acque sotterranee e idrogeotermica degli acquiferi;

- criteri ed indirizzi per gli studi idrogeologici nella progettazione di sistemi-impianti geotermici per bassissima entalpia: opere di presa (e resa) a ciclo aperto (open-loop - a pozzo - ad acqua) e chiuso (closed-loop - a sonda - a secco);

- criteri ed indirizzi per analisi quantitativa e modellazione idrogeotermica del serbatoio e geochimica dei fluidi;

- valutazione dell'impatto ambientale dello sfruttamento del sottosuolo per uso geotermico (inclusa la reiniezione dei fluidi geotermici reflui): previsione, prevenzione e mitigazione del rischio di depauperamento ed inquinamento delle falde;

- problematiche specifiche della idrogeotermia nelle fasi di studio, caratterizzazione, modellazione, progettazione, realizzazione e manutenzione delle opere, di gestione della risorsa, ed aspetti economici.

Il gruppo di lavoro dovrebbe durare tre anni (2013-2015) ed avrà la sua prima riunione in occasione del IX Convegno dei Giovani Ricercatori in Geologia Applicata, che si terrà a Napoli il 14 e 15 febbraio 2013. L'obiettivo finale sarà la stesura di linee guida per gli studi idrogeologici in ambito geotermico e la proposta di istituzione di una specifica Commissione o
Network IAH (internazionale).

L'istituzione del Gruppo di Lavoro si inserisce nell'ambito di quello che il presidente del Consiglio Nazionale dei Geologi, Gian Vito Graziano, ha chiamato "il boom della geotermia", sottolineando un forte interesse in Italia verso la geotermia anche da parte del mondo professionale dei Geologi. Infatti, a fine 2011 è stato firmato un importante accordo-quadro tra UGI (Unione Geotermica Italiana) e CNG (Consiglio Nazionale dei Geologi), per una attività congiunta, specialmente nel campo della formazione (anche in collaborazione con organismi di coordinamento europeo come il FEG, Eurogeoservice e Geo'Trainet) e della partecipazione a progetti congiunti nazionali ed europei per attività di ricerca, applicazioni sperimentali e divulgazione scientifica.

Ma il crescente interesse dei geologi e soprattutto degli idrogeologi nel campo della geotermia è evidenziato anche dai numerosi eventi, conferenze e incontri scientifici sul tema. Tra questi si segnala una apposita sessione nell'ambito del convegno "IdroVulc2013 - Acquiferi Vulcanici dell'Italia centrale: Studi idrogeologici per la soluzione di problemi gestionali" (Orvieto, 16-17 maggio 2013; http://host.uniroma3.it/laboratori/idrogeologia/idrovulc.php).

Tra le iniziative editoriali spicca Acque Sotterranee, che in questo terzo numero del 2012, esce proprio con uno speciale dedicato alla geotermia, con molti articoli di grande interesse scientifico, ma soprattutto dagli stimolanti risvolti e prospettive professionali. Oggi, in questo momento di profonda crisi economica, è indispensabile infatti per gli idrogeologi professionisti scorgere nuovi orizzonti di lavoro per il futuro, che diano opportunità di applicare le professionalità dei singoli nell'ambito di progetti energetici alternativi, di ampio respiro per il paese.

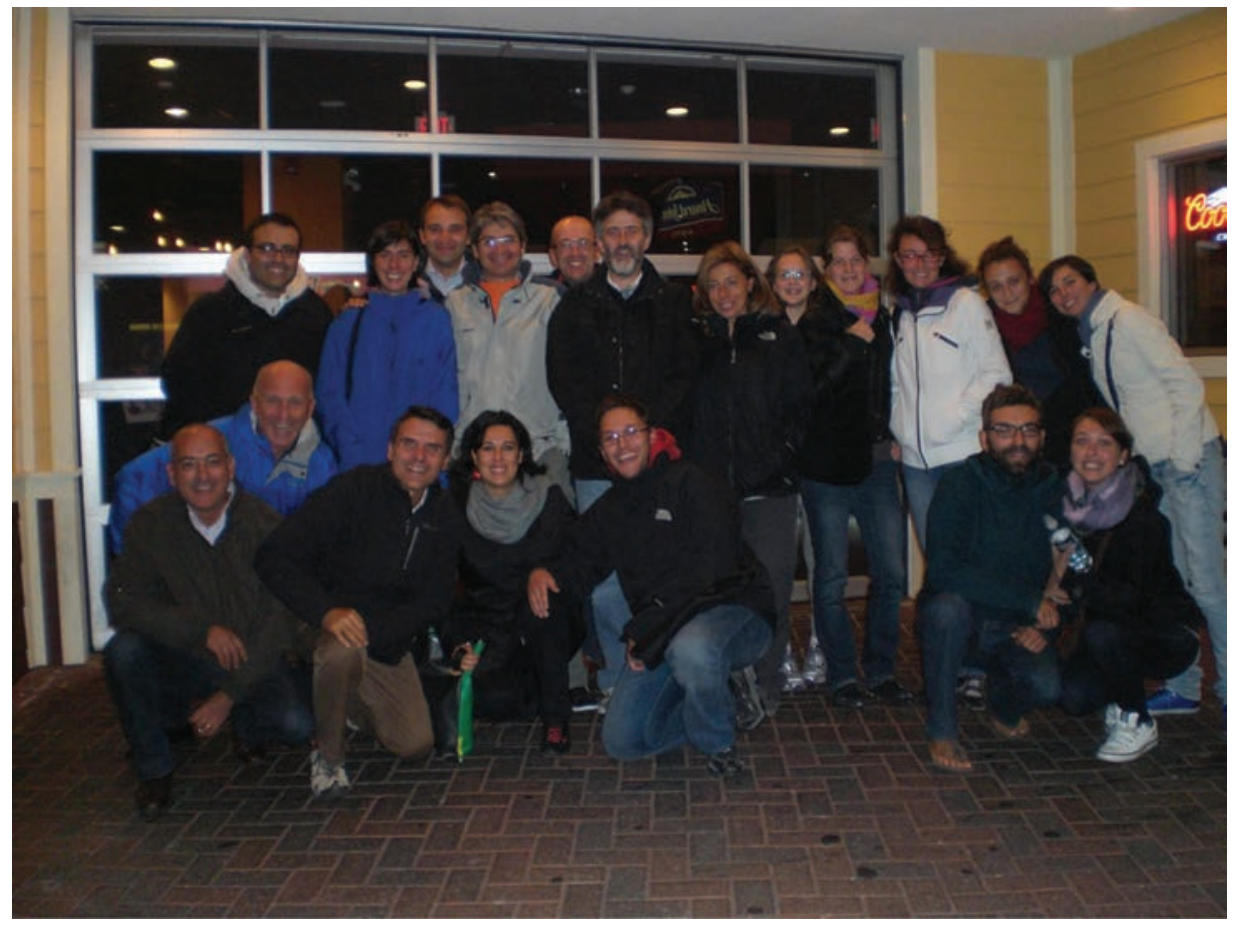

Fig. 2 -Alcuni degli italiani partecipanti al congresso di Niagara Falls (Canada) 